\title{
Ependimomas del filum terminal. Análisis de 20 casos consecutivos
}

M. Gelabert-González; A. Arcos-Algaba; R. Serramito-García; D. Castro-Bouzas; J.M. Santín-Amo; E. AranEchabe; A. Prieto-González; F.J. Bandin-Diéguez y A. García-Allut

Servicio de Neurocirugía. Hospital Clínico Universitario de Santiago de Compostela. Departamento de Cirugía. Universidad de Santiago de Compostela.

Resumen

Objetivos. Analizar las características clínicas, radiológicas y los resultados quirúrgicos de una serie de ependimomas del filum terminal.

Pacientes y método. Se estudia retrospectivamente 20 pacientes con 21 ependimomas del filum terminal tratados durante un período de 21 años (1988-2008). Todos los pacientes fueron diagnosticados con resonancia magnética e intervenidos quirúrgicamente.

Resultados. La relación varón-mujer fue de 1:1.5 y la media de edad al diagnóstico de 44.8 años (rango 1564). El primer síntoma fue dolor radicular (12 casos) y lumbalgia en los 8 restantes, con una duración media de la sintomatología antes del diagnostico de 8.7 años (rango 0.6-32). Todos los pacientes fueron intervenidos quirúrgicamente realizándose resección completa de 17 tumores y subtotal de 4 . Histológicamente 20 tumores fueron ependimomas mixopapilares (grado I ) y un caso grado II. El período de seguimiento fue de 8 años (rango 1-18).

Conclusiones. Los ependimomas del filum terminal, son tumores de crecimiento lento con una mayor incidencia en adultos jóvenes. La forma de presentación más habitual es con dolor lumbar con un largo tiempo de evolución. Aunque la mayoría son tumores de bajo grado histológico, tienen una especial tendencia a crecer y las recidivas locales no son raras.

PALABRAS CLAVE: Ependimoma. Filum terminal. Resonancia magnética. Tumor medular. Tumor mixopapilar.

Filum terminale ependymomas. Analysis of a serie of 20 consecutive cases

Summary

Objective. To analyze the clinical, radiological and surgical outcome of a series of filum terminale

Recibido: 21-12-09. Aceptado: 26-01-10 ependymomas.

Patients and methods. This retrospective study involved 20 patients with 21 ependymomas of the filum terminale encountered during a 21 year period (19882008). All patients were diagnosed using MRI and surgically treated.

Results. The male: female ratio was $1: 1.5$, and the mean age at diagnosis was 44.8 years (range 15-64). First symptom included radicular pain (12 cases) and lumbar pain in the other 8 cases, with average symptom duration of 8.7 years (range 0-6-32). All patients underwent open biopsy, seventeen tumours received gross-total resection and 4 received subtotal resection. Histologically, 20 tumours were myxopapillary ependymomas (grade I) and 1 case a grade II ependymoma. The mean follow-up period was 8 years (range 1-18 years).

Conclusions. Filum terminale ependimomas are slow growing tumours of the cauda equina with a high incidence in young adults. The most common presentation is with low back pain long time evolution. Although ependymomas of the filum terminale are thought to be benign, local recurrence is not uncommon.

KEY WORDS: Ependymoma. Filum terminale. Magnetic resonance imaging. Myxopapillary tumor. Spinal cord tumor,

\section{Introducción}

Los ependimomas medulares son tumores poco frecuentes que constituyen menos del $5 \%$ de todos los tumores del sistema nervioso central (SNC) y entre el 10-15\% de todos los tumores espinales ${ }^{14,19}$. Los ependimomas de la región lumbo-sacra representan el 30\% de todos los ependimomas espinales y se trata habitualmente de ependimomas mixopapilares, variedad descrita por Kernohan en $1932^{16}$ y que de acuerdo con la clasificación de la organización mundial de la salud (OMS) son tumores grado I. Clínicamente se caracterizan por presentarse con dolor lumbar con o sin irradiación ciática, por lo que se confun- 


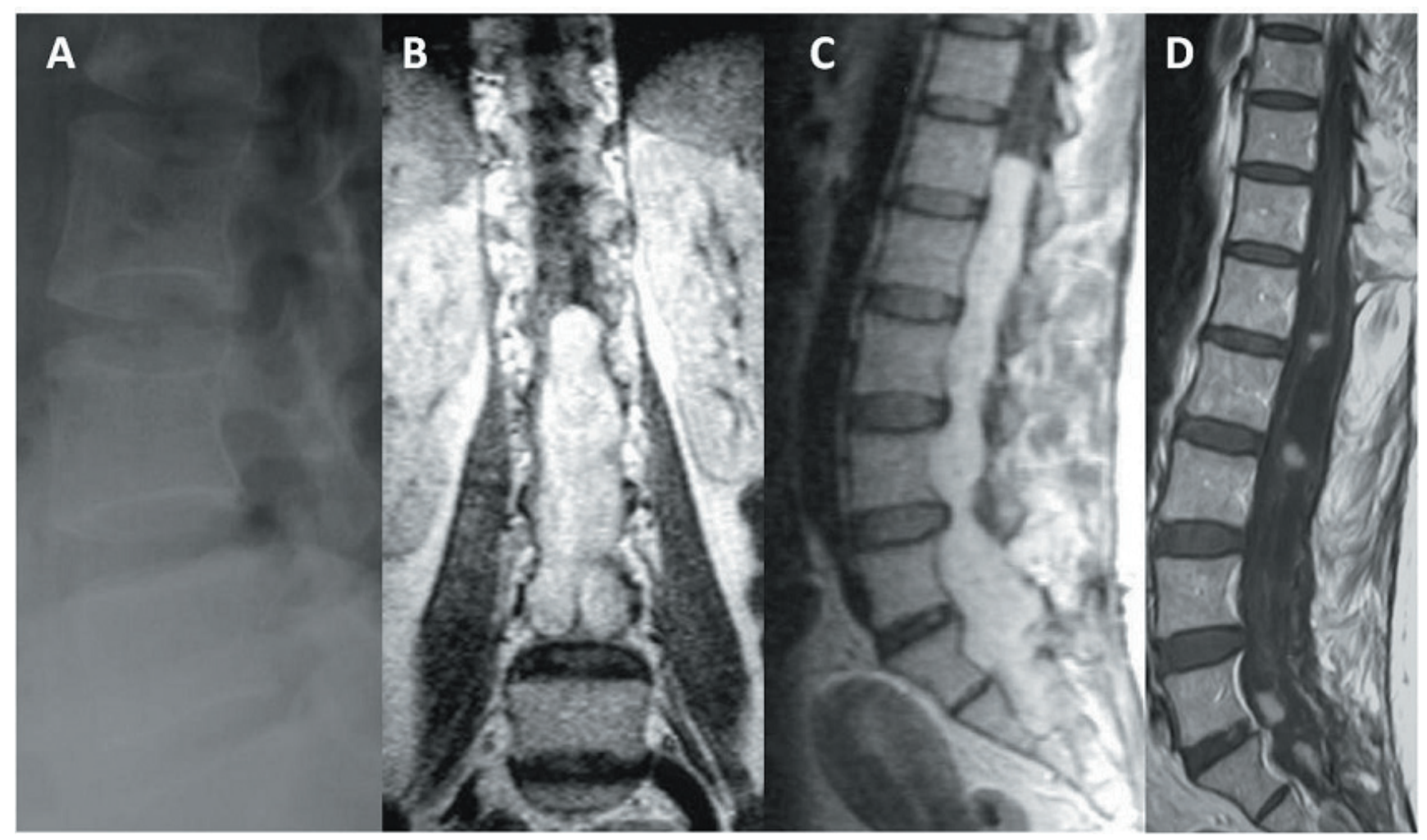

Figura 1. A: radiología simple mostrando la erosión de la cara posterior de los cuerpos vertebrales. B y C: $R M(T 1+G d)$ mostrando el voluminoso ependimoma lumbo-sacro. D: RM posoperatoria (T1 con Gd) nódulos hipercaptantes (caso 6).

den fácilmente con otros cuadros de lumbalgia, retrasando el diagnóstico durante largos periodos de tiempo ${ }^{34}$. El pronóstico tras el tratamiento quirúrgico va a depender de la situación neurológica previa y sobre todo del tamaño del tumor, ya que éste es el principal determinante para que la resección pueda ser total y la posibilidad de presentar lesiones quirúrgicas sobreañadidas.

Presentamos nuestra experiencia con 20 pacientes afectos de ependimomas localizados en la región del filum terminal en un período de 21 años y con un seguimiento mínimo de 18 meses.

\section{Pacientes y método}

Realizamos un estudio retrospectivo y descriptivo de todos los pacientes intervenidos de ependimomas del filum terminal entre Enero de 1988 y Junio de 2008 y que han sido diagnosticados empleando al menos resonancia magnética. La serie constituye el $9.4 \%$ de todos los tumores medulares intervenidos en el mismo período de tiempo.

Analizamos como datos descriptivos: edad, sexo, antecedentes personales, forma de presentación clínica y tiempo de evolución de los síntomas. Así mismo se revisaron las técnicas de diagnóstico utilizadas, hoja de intervención quirúrgica, complicaciones presentadas, análisis del informe patológico y seguimiento de los pacientes.

El estado clínico fue evaluado pre y postoperatoriamente empleando la escala de Frankel $^{10}$ (A: pérdida completa de funciones motora y sensitiva, B: solo conservada la sensibilidad, C: pérdida parcial de funciones motora y sensitiva, D: conserva función motora, E: sensibilidad y motilidad normales). Una paciente fue publicada previamente como caso clínico ${ }^{12}$.

\section{Resultados}

La serie esta formada por 12 mujeres y 8 varones con edades comprendidas entre 15 y 64 años (media 44.8), siendo de 44.3 años para las mujeres y de 45.6 para los varones. Los síntomas de inicio fueron dolor radicular (ciática) en 12 casos y lumbalgia crónica en los otros 8 pacientes. El tiempo transcurrido desde el inicio de los síntomas al diagnóstico osciló entre 0.6 y 32 años (media: 8.7). Cuatro pacientes, antes del diagnóstico del ependimoma espinal, fueron intervenidos quirúrgicamente en la misma región anatómica, de hernia discal de localización lumbar en tres casos y al otro paciente se le había realizado una artrodesis $\mathrm{L}_{2}-\mathrm{S}_{1}$ en otro centro (Tabla I).

Exploración: la exploración clínica mostraba disfunciones motoras en 7 casos aunque existían alteraciones 


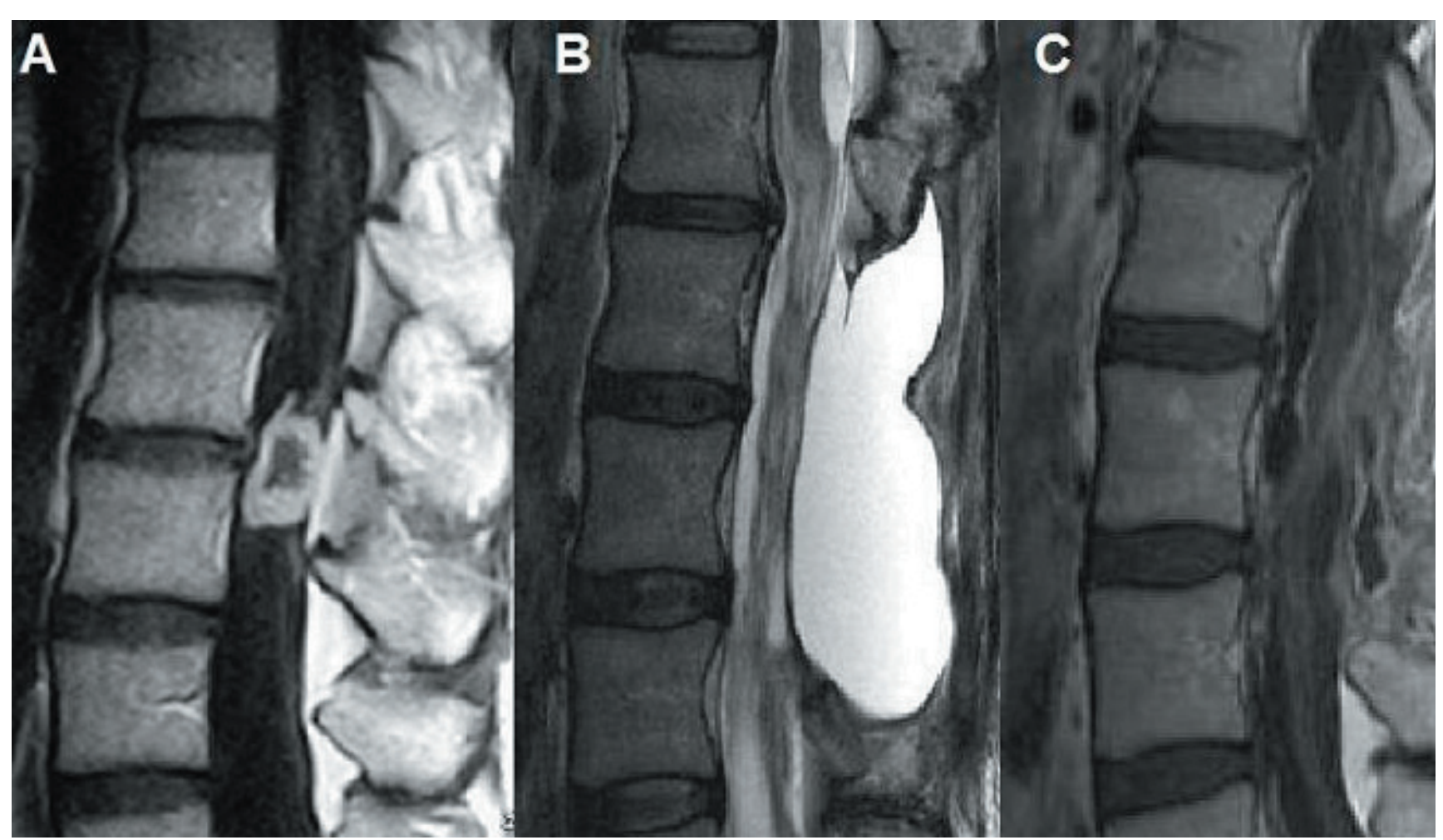

Figura 2. $A: R M(T 1+G d)$ ependimoma con captación periférica de contraste localizado entre $L_{2}-L_{3}$. $B: R M(T 2)$ realizada al mes de la cirugía mostrando un voluminoso pseudomeningocele. C: RM (T1) realizada a los 6 meses de la cirugía mostrando la resolución del pseudomeningocele.

de reflejos osteotendinosos en otros 4 pacientes. Las alteraciones sensitivas afectando a los últimos dermatomas lumbares y sacros se objetivaron 6 casos. De acuerdo con la escala de Frankel 11 pacientes se encontraban en grado E, sin existir ninguno en grados A o B.

Radiología simple: se realizó radiología simple lumbar en 14 casos, observándose en cuatro de ellos erosión en la cara posterior de los cuerpos vertebrales (scalloping), siendo normal en el resto (Figura 1).

Resonancia magnética (RM): en todos los casos se observó una masa intradural con un tamaño que oscilaba entre 1 y $14 \mathrm{~cm}$ (media $4.6 \mathrm{~cm}$ ), encontrándose el tumor entre los segmentos vertebrales $\mathrm{L}_{1}$ y $\mathrm{S}_{3}$. La lesión más pequeña era inferior al tamaño de un cuerpo vertebral y la de mayor tamaño ocupaba 8 cuerpos. La localización más frecuente por cuerpos vertebrales era a nivel $\mathrm{L}_{3}$ (16 casos) seguida por $\mathrm{L}_{2}$ (14 casos). El canal espinal se encontraba agrandado axialmente en 8 pacientes y en 2 casos se observaba extensión del tumor fuera del canal espinal. Diecinueve pacientes tenían tumor único y otro presentaba 2 tumores adyacentes. Las características de señal mostraban que en T1 el tumor era isointenso en 20 tumores e hiperintenso en otro. En secuencia T2 todos los ependimomas eran hiperintensos. En todos los casos existía captación de contraste, que era heterogéneo en 15 tumores y homogéneo en los otros 6 (Figura 2).

Tratamiento: todos los pacientes fueron intervenidos mediante una laminectomía centrada sobre el tumor. Empleando técnica microquirúrgica se realizó extirpación completa de 17 tumores y parcial en los otros 4 casos. En cinco casos se utilizó monitorización neurofisiológica de las raíces lumbo-sacras. Se presentaron 3 casos de fístula de LCR que precisaron una reintervención para el cierre de la misma, otro paciente presentó un pseudomeningocele que cedió espontáneamente. Tras la cirugía 3 pacientes empeoraron su escala de Frankel en un grado, que se recuperaron a los 12 y 16 meses en 2 casos, permaneciendo el otro en el grado inferior (caso 6). En un caso de extirpación incompleta se realizó nueva cirugía a los 2 años (caso 4) sin lograrse la exéresis completa. Ninguno de los pacientes recibió tratamiento complementario con radioterapia o quimioterapia.

Patología: los 21 tumores fueron estudiados histopatologicamente, diagnosticándose en 20 casos un ependimoma mixopapilar típico (grado I de la OMS) y el otro tumor correspondía a un ependimoma grado II de la OMS (Figura $3)$.

Evolución: el tiempo medio de seguimiento fue de 8 años (rango 1.5-18) y, a excepción de dos pacientes cuyo control perdimos a los 2 y 4 años, y el más antiguo de la 


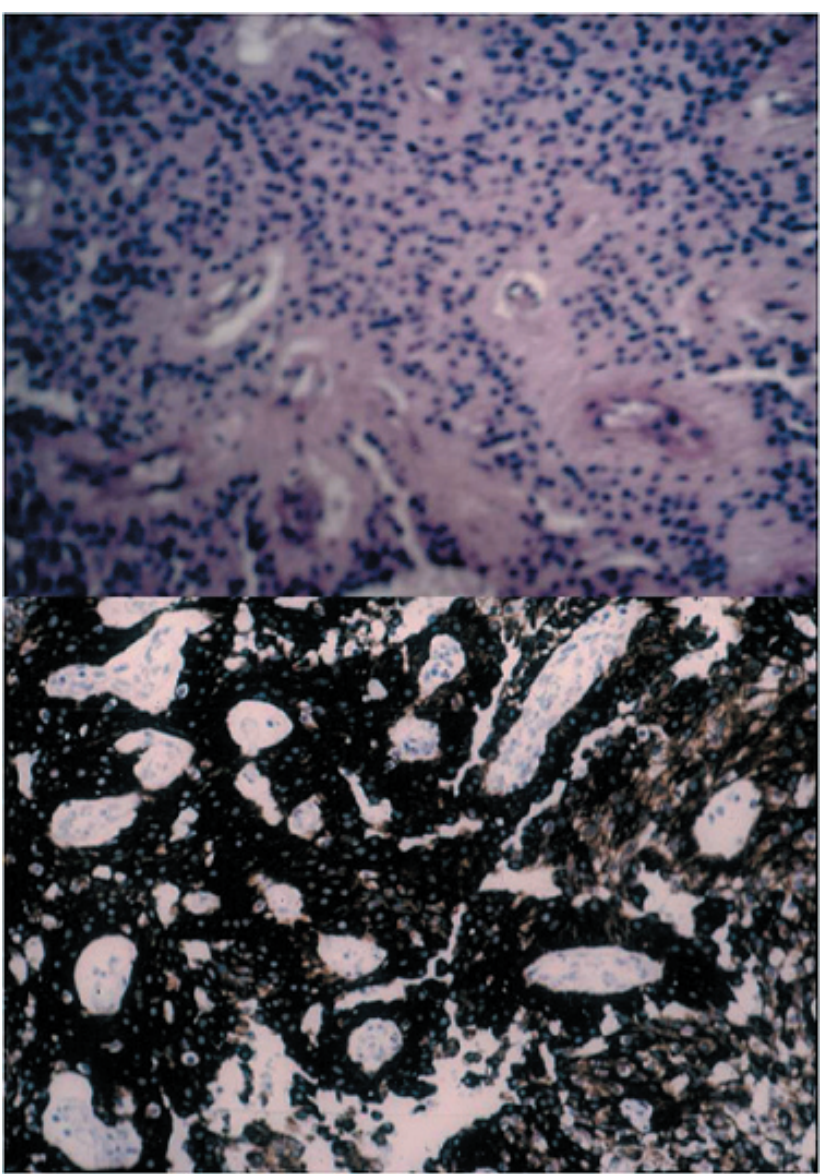

Figura 3. Superior: imagen característica de un ependimoma mixopilar en la que se pueden visualizar las seudorosetas perivasculares (H.E. 200x). Inferior: positividad intensa del tumor para PGFA (100x).

serie que falleció a los 12 años, todos siguen controles periódicos. Las evaluaciones de imagen con RM muestran que no hay restos tumorales en 15 pacientes. Los casos 6 y 10 presentan pequeños nódulos hipercaptantes en RM pero al mantenerse una estabilidad clínica y radiológica se decide una actitud expectante. La supervivencia libre de enfermedad a los 5 y 10 años para los pacientes con este seguimiento mínimo fue del $80 \%$ y la supervivencia global del $100 \%$.

\section{Discusión}

Los ependimomas del filum terminal representan entre el $1-2 \%$ de todos los tumores medulares y se han descrito prácticamente en todas las edades. En los adultos llegan a representar entre el 50-60\% de todos los tumores medulares, mientras que en el niño solo constituyen el 10$20 \%{ }^{18,20,39,49}$.

La forma de presentación de los ependimomas lumbo- sacros no difiere de la de otros tumores de la misma localización, la mayoría debutan con un cuadro de dolor lumbar con o sin irradiación a miembros inferiores, que en la mayoría de las ocasiones anteceden períodos prolongados de tiempo antes del diagnóstico ${ }^{20,34}$. Otros síntomas habituales son parestesias en miembros inferiores y alteraciones esfinterianas $^{20}$. En la literatura existen numerosas referencias en relación con distintas formas de presentación en los ependimomas de esta localización: como hemorragias intratumorales espontáneas o traumáticas ${ }^{2,25}$, hemorragia subaracnoidea $^{35}$, el desarrollo de una hidrocefalia comunicante ${ }^{42}$, probablemente en relación con microsangrados asintomáticos o al incremento de las proteínas en el líquido cefalorraquídeo, como ya ha sido publicado en otros tumores medulares.

La duración de la sintomatología previa al diagnóstico se ha reducido progresivamente desde la introducción de la $\mathrm{RM}$ en el diagnostico de la patología raquimedular. Así en los casos publicados hasta 1990 la duración de los síntomas era en general superior a 60 meses, tanto en adultos como en pacientes pediátricos ${ }^{30,32}$. En las series posteriores a 1990 este período se reduce de forma significativa hasta los 12-36 meses ${ }^{6,8,34}$. En nuestra experiencia, aunque todos los pacientes han sido diagnosticados con RM, los 10 primeros casos tenían un tiempo de evolución de la sintomatología de 12.8 años, siendo en los 10 últimos de 4.6.

Aunque en nuestra serie hay un caso de ependimoma grado II, la mayoría de los ependimomas de esta localización son de tipo mixopapilar, originándose en células ependimarias del filum y se incluyen dentro del grado I de la $\mathrm{OMS}^{3}$. Excepcionalmente pueden localizarse en el espacio extradural probablemente en vestigios medulares del cóccix en la porción distal del tubo neural ${ }^{5,42,44}$. Macroscópicamente son tumores blandos, lobulados, que no invaden el parénquima circundante y en el estudio histológico se trata de tumores formados por células que se disponen formando papilas alrededor de un estroma conectivo con abundante degeneración mixoide ${ }^{44}$. Las células, se disponen en torno a centros conectivos vascularizados que ofrecen la imagen de una zona fibrilar en torno al vaso; no es infrecuente observar la presencia de microhemorragias o calcificaciones. Desde el punto de vista inmunohistoquímico las células muestran positividad a la proteína gliofibrilar ácida (PGFA). Estudios recientes han podido observar un alto índice de expresión de la prostaglandina COX-2 y del factor de crecimiento endotelial (VEGF) en los ependimomas espinales y con mayor incidencia en los de estirpe mixopapilar ${ }^{24}$; de confirmarse en posteriores estudios esta expresión, podría dar lugar en el futuro a una nueva modalidad de tratamiento mediante inhibidores específicos de la angiogénesis tumoral ${ }^{23}$. En la literatura se han descrito otras variedades histológicas de ependimomas del filum como subependimomas, 
ependimomas celulares, de células gigantes ${ }^{50}$, tanacíticos ${ }^{40} \mathrm{O}$ ependimomas grado $\mathrm{II}^{20}$.

Los estudios citogenéticos de los ependimomas han demostrado alteraciones en el cromosoma 22, en forma de monosomía, delecciones o translocaciones; otros cromosomas afectados son el $9,10,13$ y $17^{29}$. No se ha podido observar relación con factores carcinogénicos, ambientales o hereditarios ${ }^{3,22}$.

La radiología simple no es una herramienta habitual en el diagnóstico actual de los tumores medulares. En los casos de mayor volumen y más evolucionados se puede ver erosión en los pedículos y la cara posterior de los cuerpos vertebrales, como ocurría en 4 de nuestros pacientes.

Aunque en la resonancia magnética no existen unas características específicas para los ependimomas del filum, constituye la prueba diagnóstica de elección ya que permite conocer la extensión del tumor, su relación con las estructuras medulares y radiculares y evaluar la posible existencia de diseminación en el espacio subaracnoideo. Únicamente uno de nuestros pacientes presentaba más de un tumor, sin embargo en la literatura entre el $10-43 \%$ de los pacientes presentan más de un ependimoma en el momento del diagnóstico, tratándose posiblemente de metástasis a través del $\mathrm{LCR}^{7,12,47}$.

Las características de resonancia no son específicas y típicamente son isointensos en relación con la médula espinal en secuencias $\mathrm{T} 1$ e hiperintensos en secuencias $\mathrm{T} 2$. En ocasiones pueden ser hiperintensos tanto en $\mathrm{T} 1$ como en $\mathrm{T} 2$ reflejando un alto contenido en mucina o por la presencia de focos hemorrágicos ${ }^{17,46}$. Se realzan con contraste de manera homogénea ( $75 \%$ de casos) o heterogénea $(25 \%)$ y con una intensidad entre moderada y alta. Para Wippold et $\mathrm{al}^{47}$ los tumores con realce homogéneo tienden a ser bien encapsulados, mientras que los heterogéneos no tienen cápsula. Aunque la RM no es idónea para el estudio de la cortical del hueso, es posible visualizar la erosión ósea así como la expansión del canal espinal y de los agujeros de conjunción ${ }^{47}$.

El diagnóstico diferencial debe hacerse con otros tumores de la misma localización, como astrocitomas, paragangliomas del filum ${ }^{13}$, schwannomas ${ }^{4,6}$, epidermoides, etc. El tratamiento microquirúrgico es el de elección en los tumores de esta localización. En ependimomas de gran tamaño, la extirpación se ve complicada por la existencia de conexiones con el cono medular o las raíces de la cola de caballo. Su manipulación puede ocasionar lesiones neurológicas sobreañadidas. Cuando existe buen plano de disección la resección completa es posible sin necesidad de aplicar tratamientos posteriores ${ }^{3}$. Aunque en nuestra experiencia, únicamente se ha utilizado monitorización neurofisiológica en 4 casos, pensamos, al igual que otros autores, que el empleo de potenciales evocados somatosensoriales y motores, electromiografía de los músculos de miembros inferiores así como de los esfínteres anal y uretral, contribuyen a obtener un estricto control funcional del cono medular y del filum terminal, permitiendo exéresis más amplias y seguras, reduciendo de forma significativa las secuelas neurológicas ${ }^{9,36}$.

A pesar de tratarse de procesos de baja malignidad existe una alta tasa de recidivas y de diseminación del tumor por el espacio subaracnoideo. Rezai et al. ${ }^{31}$ encontraron que los ependimomas mixopapilares tiene hasta 3.6 veces más posibilidades de sufrir una diseminación en comparación con otros tumores de bajo grado.

No existe consenso de actuación en los casos de recidiva tanto se haya realizado una resección completa o parcial. El papel de la radioterapia es controvertido. Ross y Rubinstein $^{33}$ en una revisión sobre 131 casos de ependimomas mixopapilares no encuentran diferencias importantes entre los casos radiados y los no radiados después de resección completa o incompleta ${ }^{46}$. Sin embargo, numerosas series sugieren que la radiación es el tratamiento indicado en casos de resecciones incompletas, debido a la alta tasa de recidivas, aunque en muchas de estas series, aparecen mezclados ependimomas del filum con ependimomas puramente intramedulares (grados II) o incluso intracraneales. En la revisión de Schild et $\mathrm{al}^{38}$ sobre 80 ependimomas medulares tratados en la Clínica Mayo, de los cuales 12 eran mixopapilares, todos, independientemente del grado de resección, fueron sometidos a radioterapia con dosis entre 40 y 59.6 Gy; a los 5 años, el control local de los pacientes que recibieron más de 50 Gy fue del $100 \%$, siendo del $67 \%$ en aquéllos que recibieron menos de los $50 \mathrm{~Gy}$, concluyendo que existe una relación directa entre la dosis administrada y el control tumoral ${ }^{37,38}$.

Por el contrario Wahab et al. ${ }^{47}$ en una serie de 22 pacientes con ependimoma (únicamente 9 grados I) no puede establecer una relación dosis-respuesta para el control tumoral, aunque en aquellos tumores menores de $6 \mathrm{~cm}$ el control tumoral es mejor que en los mayores, la supervivencia libre de progresión es de $92 \%$ contra $58 \%$ a los 10 años.

Las dosis por encima de los 55 Gy aumentan de forma significativa el riesgo de mielopatía, por lo que en la mayoría de los centros se aplican dosis por debajo de esta cifra $^{21,47}$.

A pesar de tratarse de un tumor de bajo grado, no es infrecuente que se diseminen dentro del neuroeje, tanto a nivel espinal como cerebral ${ }^{23,27,28}$, que pueden obligar en algunas ocasiones a realizar radioterapia craneoespinal ${ }^{8}$. Excepcionalmente se han descrito casos de extensión fuera del $\mathrm{SNC}^{15}$.

Numerosas publicaciones analizan los diversos factores pronósticos de este tipo de tumores. Seha encontrado relación significativa con edad del paciente ${ }^{1,}$, estado clínico ${ }^{26}$, afectación de esfínteres ${ }^{38}$, duración de los síntomas ${ }^{6,11,26}$, localización ${ }^{26}$, características de imagen con $\mathrm{RM}^{34}$, presencia o no de 


\begin{tabular}{|c|c|c|c|c|c|c|c|c|c|c|c|c|c|c|c|c|c|c|c|c|c|}
\hline & 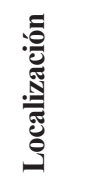 & $\begin{array}{l}0 \\
\hat{1} \\
\stackrel{1}{a}\end{array}$ & 声 & 志 & 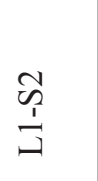 & 今3 & 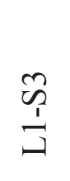 & $\begin{array}{l}\vec{\sim} \\
\stackrel{\Lambda}{J} \\
\end{array}$ & 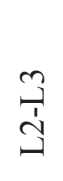 & 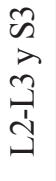 & $\stackrel{9}{\rightrightarrows}$ & 声 & 声 & 光 & $\begin{array}{l}\overrightarrow{\tilde{n}} \\
\hat{n} \\
\end{array}$ & $\underset{3}{\stackrel{3}{3}}$ & 先 & $\frac{3}{3}$ & 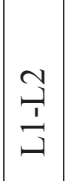 & $\mathcal{J}$ & 今. \\
\hline & 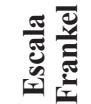 & $U$ & 工 & 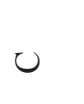 & $U$ & 山 & 디 & U & 山 & 龱 & U & O & 띠 & D & 피 & 山 & D & $U$ & 山 & I & 피 \\
\hline 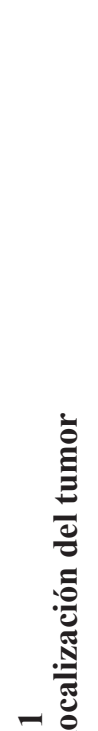 & 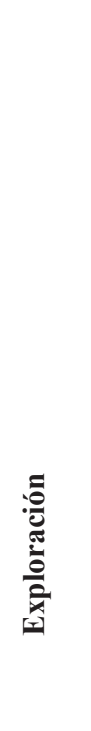 & 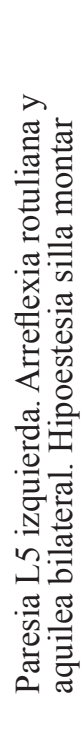 & 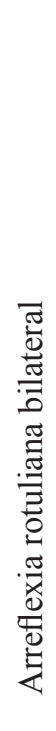 & 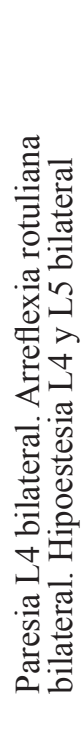 & 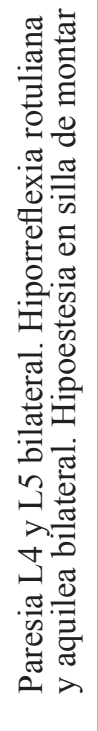 & 节 & 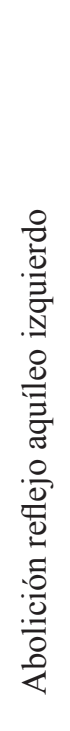 & 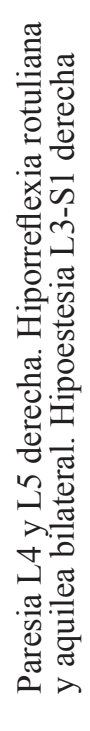 & $\begin{array}{l}\overline{\widetilde{I}} \\
\text { Eे } \\
\text { Z }\end{array}$ & 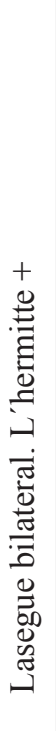 & 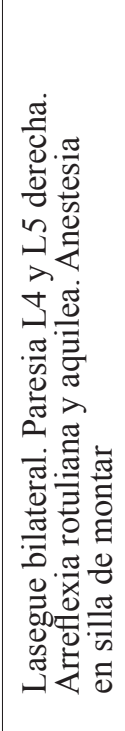 & 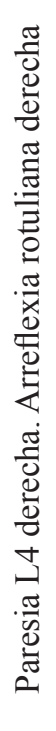 & 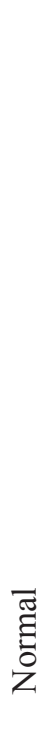 & 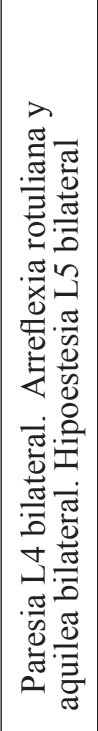 & 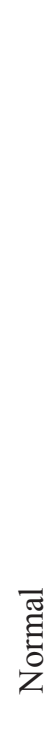 & 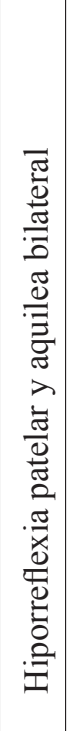 & 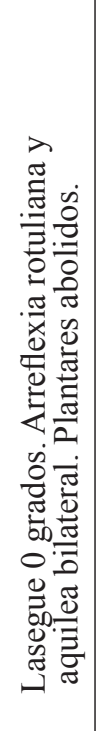 & 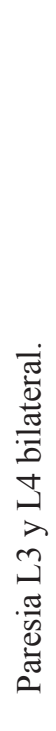 & $\begin{array}{l}\bar{\pi} \\
\tilde{\Xi} \\
z \\
z\end{array}$ & $\begin{array}{l}\bar{\Xi} \\
\tilde{\Xi} \\
z\end{array}$ & 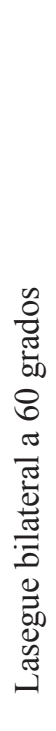 \\
\hline
\end{tabular}

需

跣

נ.

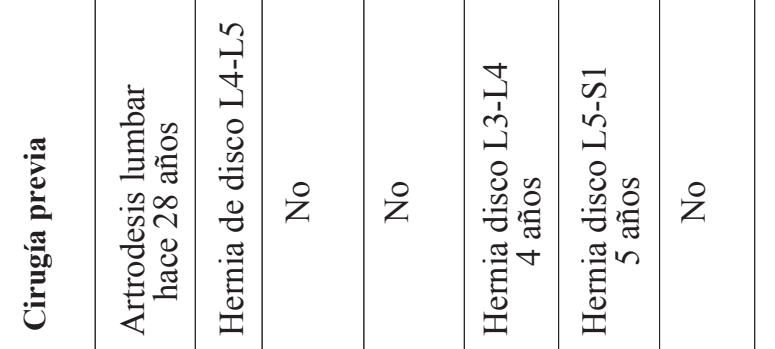

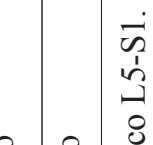

ì

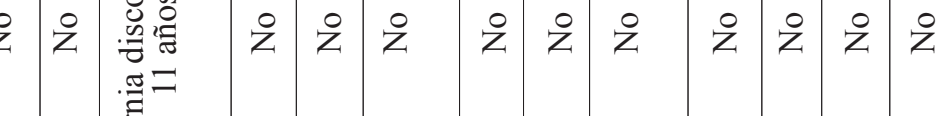
苞

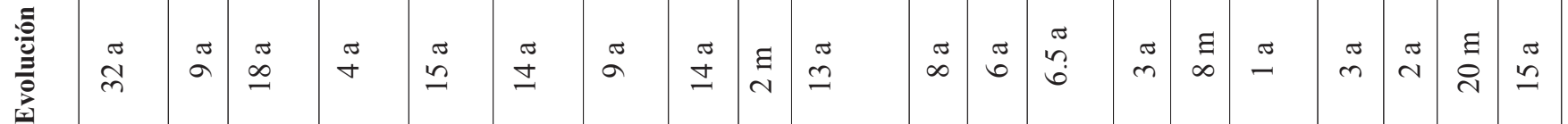

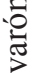
$\ddot{>}$

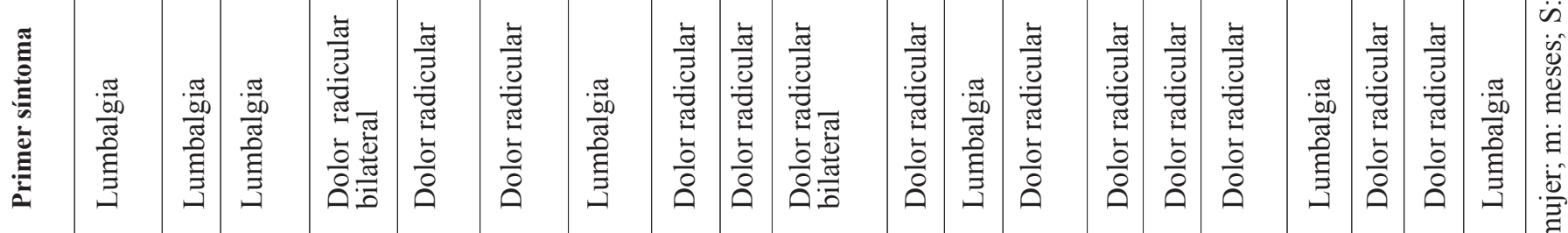

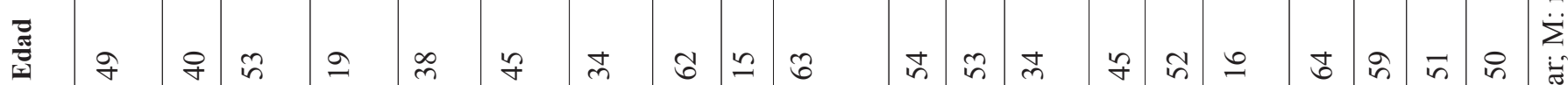

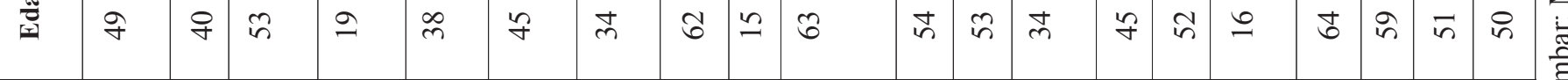

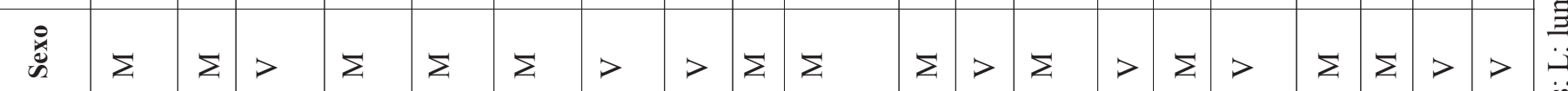

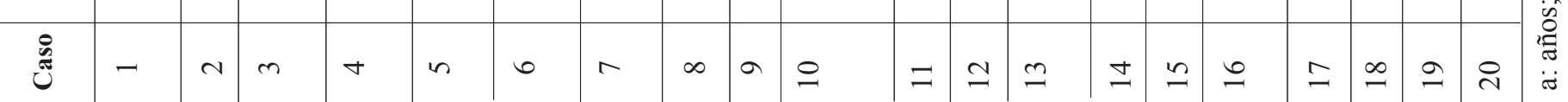




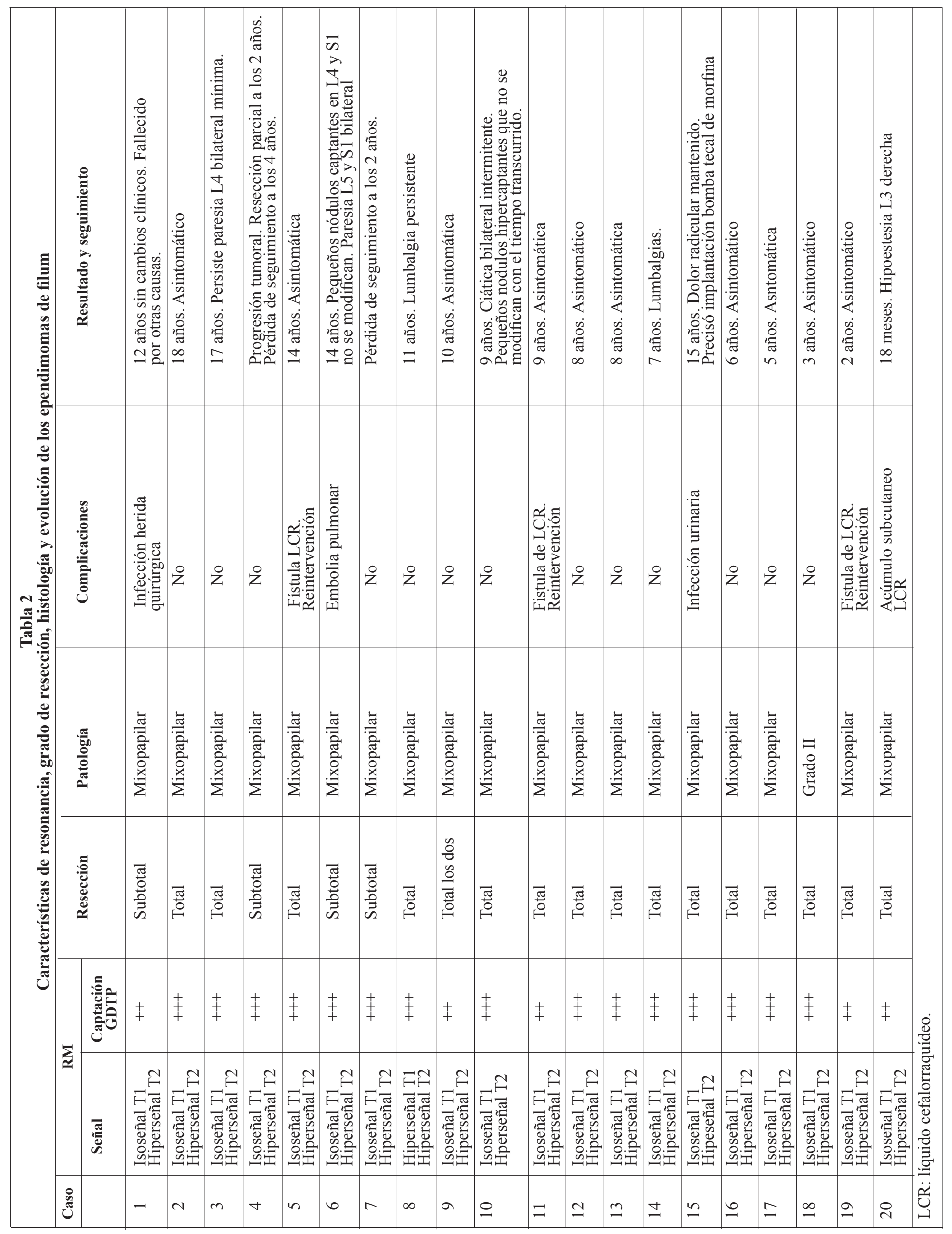


cápsula tumoral ${ }^{34}$, grado de resección tumoral ${ }^{23,34}$, tratamiento inicial y dosis de radioterapia ${ }^{1}$. Únicamente, Akyurek et $\mathrm{al}^{1}$ en una serie de 35 pacientes encuentran como factores significativos de buen pronóstico la edad de los pacientes $(<35$ contra $>35$ años $)$ y la modalidad de tratamiento inicial (cirugía contra cirugía y radioterapia).

\section{Bibliografía}

1. Akyurek, S., Chang, E.L., Yu, T.K., et al.: Spinal myxopapillary ependymoma outcomes in patients treated with surgery and radiotherapy at M.D. Anderson Cancer Center. J Neurooncol 2006; 80: 177-183.

2. Argyropoulou, P.I., Argyropoulou, M.I., Tsampoulas, C., Gogos, P., Manavis, I., Efremidis, S.C.: Myxopapillary ependymoma of the conus medullaris with subarachnoid haemorrhage: MRI in two cases. Neuroradiology 2001; 43: 489-491.

3. Bagley, C.A., Wilson, S., Kothbauer, K.F., Bokland, M.J., Epstein, F., Jallo, G.I.: Long term outcomes following surgical resection of myxopapillary ependymomas. Neurosurg Rev 2009; 32: 321-334.

4. Bavbek, M., Altinörs, M.N., Caner, H.H., Bilezikçi, B., Aðildere, M.: Lumbar myxopapillary ependymoma mimicking neurofibroma. Spinal Cord 2001; 39: 449-452.

5. Castro-Boix, S., Coronado-Llanos, D., Pradell, J., Nadal, A.: Ependimoma mixopapilar de la región sacra. Cir Esp 2007; 82: 313-314

6. Cervoni, L., Celli, P., Cantore, G., Fortuna, A.: Intradural tumors of the cauda equina: a single institution review of clinical characteristics. Clin Neurol Neurosurg 1995; 97 : 8-12.

7. Chan, H.S.L., Becker, L.E., Hoffman, H.J., et al.: Myxopapillary ependymoma of the filum terminale and cauda equina in childhood: report of seven cases and review of the literature. Neurosurgery 1984; 14: 204-210.

8. Chinn, D.M., Donaldson, S.S., Dahl, G.V., et al.: Management of children with metastatic spinal myxopapillary ependymoma using craniospinal irradiation. Med Ped Oncol 2000; 35: 443-445.

9. Collado-Corona, M.A., de Leo-Vargas, R., SandovalSánchez, V., Díaz-Hernández, A., Gutiérrez-Sougarret, J., Shkurovich-Bialik, P.: Vigilancia neurológica transoperatoria multimodal en cirugía de columna. Cir Cir 2009; 77: 385 390.

10. Frankel, H.L., Hancock, D., Hyslop, G., et al.: The value of postural reduction in the intial management of closed injuries of the spine with paraplegia and tetraplegia. Paraplegia $1969 ; 24: 179-192$.

11. Gavin, D., Farooqi, N., Pigott, T.J.D., et al.: Outcome predictors in the management of spinal cord ependymoma. Eur Spine J 2007; 16: 399-404.

12. Gelabert-González, M., Prieto, A., Abdulkader, I.,
Cutrín, J.M.: Double ependymoma of the filum terminale. Childs Nerv Syst 2001; 17: 106-108

13. Gelabert-González, M.: Paragangliomas of the lumbar region. J Neurosurg Spine 2005; 2: 354-365.

14. Gelabert-González, M.: Tumores medulares primarios. Análisis de una serie de 168 pacientes. Rev Neurol 2007; 44: 269-274.

15. Graf, M., Blaeker, H., Otto, H.F.: Extraneural metastasizing ependymoma of the spinal cord. Case report. Pathol Res Oncol 1999; 5: 56-60.

16. Kernohan, J.W.: Primary tumors of the spinal cord and intradural filum terminale. En: Penfield W (ed). Cytology and cellular pathology of the nervous system. Vol 3, New York: Paul B. Hoeber, 1932, pp. 993-1035.

17. Koeller, K.K., Rosenblum, R.S., Morrison, A.L.: Neoplasms of the spinal cord and filum terminale: RadiologicPathologic correlation. RadioGraphics 2000; 20: 1721-1749.

18. Kumar, V., Shankar, R.: Myxopapillary ependymoma of the filum terminale. Pediatr Radiol 2009; 39: 415.

19. López-Escobar, M., Gómez, E., Contreras, A., Ramos, C.: Ependimomas del filum terminal. Neurocirugía 1998; 9: 11-20.

20. Meneses, M.S., Leal, A.G., Periotto, L.B., et al.: Primary filum terminale ependymoma. A series of 16 cases. Arq Neuropsquiatr 2008; 66: 529-533.

21. McCunniff, A.J., Liang, M.J.: Radiation tolerance of the cervical spinal cord. Int J Radiat Oncol Biol Phys 1986; 12: $1765-1769$.

22. Moynihan, T.J.: Ependymal tumors. Curr Treat Options Oncol 2003; 4: 517-523.

23. Nakamura, M., Ishii, K., Watanabe, K., Tsuji, T., Matsumoto, M., Toyama, Y., Chiba, K.: Long-term surgical outcomes for myxopapillary ependymomas of the cauda equina. Spine 2009; 34: E756-E760.

24. Naruse, T., Matsuyama, Y., Ishiguro, N.: Cyclooxygenase-2 expresssion in ependymoma of the spinal cord. J Neurosurg Spine 2007; 6: 240-246.

25. Ozdemir, O., Calisaneller, T., Coven, I., Altinors, N.: Posttraumatic intratumoral haemorrhage: an unusual presentation of spinal ependymoma. Eur Spine J 2007; 16 (Suppl 3): S293-S295.

26. Pica, A., Miller, R., Villá, S., et al.: The results of surgery, with or without radiotherapy, for spinal myxopapillary ependymoma: a retrospective study from the rare cancer network. Int J Radiation Onclology Biol Phys 2009; 74: 11141120.

27. Piquer, J., Riesgo, P., Navarro, J., Cabanes, J., Orozco, M., Álvarez, J.: Metástasis cerebrales del ependimoma medular: A propósito de tres casos. Neurocirugía 1996; 7: 129-132.

28. Plans, G., Brell, M., Cabiol, J., Villa, S., Torres, A., Acebes, J.J.: Intracranial retrograde dissemination in filum terminale myxopapillary ependymomas. Acta Neurochir (Wien) 2006; 148: 343-346. 
29. Quinones-Hinojosa, A., Gulati, M., Schmidt, M.H.: Intramedullary spinal cord tumors. En: Gupta N, Banerje A, Haas-Kogan D, eds. Pediatric CNS tumors. Berlin; Springer, 2004, pp.167-182.

30. Rawlinson, D.G., Herman, M.M., Rubinstein, L.J.: The fine structure of myxopapillary ependymoma of the filum terminale. Acta Neuropathol 1973; 25:1-13.

31. Rezai, A.R., Woo, H.H., Lee, M., et al.: Disseminated ependymomas of the central nervous system. J Neurosurg 1996; 85: 618-624.

32. Rivierez, M., Oueslati, S., Philippon, J., Pradat, P., et al.: Les épendymomes du filum terminal intra-dural chez l'adulte. Neurochirurgie 1990; 36: 96-107.

33. Ross, G.W., Rubinstein, L.J.: Lack of histopathological correlation of malignant ependymomas with postoperative survival. J Neurosurg 1989; 70: 31-36.

34. Sakai, Y., Matsuyama, Y., Katayama, Y., et al.: Spinal myxopapillary ependymoma. Spine 2009; 15: 1619-1624.

35. Sa'adah, M., Al Shunnar, K., Saadah, L., Shogan, A., Inshas, J., Afifi, H.: Atypical presentations of conus medullaris and filum terminale myxopapillary ependymomas. J Clin Neurosc 2004; 11: 268-272.

36. Sala, F., Bricolo, A., Faccioli, F., Lanteri, P., Gerosa, M.: Surgery for intramedullary spinal cord tumors: the role of intraoperative (neurophysiological) monitoring. Eur Spine J 2007; 16 (Suppl 2): S130-139.

37. Schild, S.E., Wong, W., Nisi, K.: In regard to the radiotherapy of myxopapillary ependimomas. Int J Radiat Oncol Biol Phys 2002; 53: 787.

38. Schild, S.E., Nisi, K., Scheithauer, B.W. et al.: The results of radiotherapy for ependymomas: the Mayo Clinic experience. Int J Radiat Oncol Biol Phys 1998; 42: 953-958.

39. Schweitzer, J.S., Batzdorf, U.: Ependymoma of the cauda equina region: diagnosis, treatment, and outcome in 15 patients. Neurosurgery 1992; 30: 202-207.

40. Shintaku, M., Sakamoto, T.: Tanycytic ependymoma of the filum terminale with pleomorphic giant cells. Brain Tumor Pathol 2009; 26: 79-82.

41. Turgut, M., Ak, H., Özara, E.: Filum terminale ependymoma with intratumoral and spinal subarachnoid hemorrhage. Surg Neurol 2006; 66: 646-647.
42. Tzekov, C., Naydenov, E., Kalev, O.: Ependymoma of the cauda equina starting with communicating hydrocephalus: a case report. Pediatr Neurosurg 2007; 43: 399-402.

43. Vaquero, J., Ratia, T., Ruiz, A., Zurita, M., Coca, S.: Ependimoma subcutáneo de la región sacro-coxígea. Neurocirugía 1997; 8: 127-129.

44. Vaquero, J.: Ependimomas de la médula espinal. En: Campos JM, Vaquero J. eds. Tumores raquimedulares. Madrid: Ramón Areces, 2007; pp. 213-230.

45. Vara-Thorbeck, R., Sanz-Esponera, J.: Intrasacral ependymoma. J Neurosurg 1970; 32: 589-592.

46. Wahab, S.H., Simpson, J.R., Michalski, J.M., Mansur, D.B.: Long term outcome with post-operative radiation therapy for spinal canal ependymoma. J Neurooncol 2007; 83: 85-89.

47. Wippold, F.J. II, Smirniotopoulos, J.G., Moran, C.J., Suojanen, J.N., Vollmer, D.G.: MR imaging of myxopapillary ependymoma: findings and value to determine extent of tumor and its relations to intraspinal structures. AJR Am J Roentgenol 1995; 165: 1263-1267.

48. Wight, D.G., Holley, K.J., Finbow, J.A.: Metastasizing ependymoma of the cauda equina. J Clin Pathol 1972; 26: 929935.

49. Yoshii, S., Shimizu, K., Ido, K., Nakamura, T.: Ependymoma of the spinal cord and the cauda equine region. $\mathrm{J}$ Spinal Disord 1999; 12: 157-161.

50. Zec, N., De Girolami, U., Schofield, D.E., Scott, R.M., Anthony, D.C.: Giant cell ependymoma of the filum terminale. A report of two cases. Am J Surg Pathol 1996; 20: 1091-1101.

Gelabert-González, M.; Arcos-Algaba, A.; SerramitoGarcía, R.; Castro-Bouzas, D.; Santín-Amo, J.M.; AranEchabe, E.; Prieto-González, A.; Bandin-Diéguez, F.J.; García-Allut, A.: Ependimomas del filum terminal. Análisis de 20 casos consecutivos. Neurocirugía 2101; 21: 381-389.

Correspondencia: Miguel Gelabert-González. Servicio de Neurocirugía. Hospital Clínico. A Choupana s/n. 15706 Santiago de Compostela. miguel.gelabert@usc.es 